\title{
Formation of the adaptive fuzzy model of the rock geological structure for exploratory drilling
}

\author{
Vitalii Tron ${ }^{1 *}$, Oleksandr Tsokurenko ${ }^{2}$, Dmytro Paraniuk ${ }^{3}$, and Iryna Haponenko ${ }^{2}$ \\ ${ }^{1}$ Kryvyi Rih National University, Department of Automation, Computer Science and Technology, \\ 11 Matusevycha St., 50027 Kryvyi Rih, Ukraine \\ ${ }^{2}$ Kryvyi Rih National University, Research Department, 11 Matusevycha St., 50027 Kryvyi Rih, \\ Ukraine \\ ${ }^{3}$ PJSC “ArcelorMittal Kryvyi Rih", Department of Security, 1 Kryvorizhstali St., 50000 Kryvyi Rih, \\ Ukraine
}

\begin{abstract}
The authors suggest an approach aimed at improving the technology, increasing energy efficiency and quality of controlling borehole drilling in mining various mineralogical-technological types of iron ore through applying a geological structure model based on data of measuring drilling parameters of a drilling rig (drilling rate, torque, energy characteristics) and rock properties on the basis of studying regularities of changing parameters of the specifically formed ultrasonic field. In rapidly changing conditions of borehole drilling, it is offered to apply a two-level adaptive optimization strategy implying a simultaneous investigation into drilling and improvement of control over this process by means of the adaptive fuzzy system.
\end{abstract}

\section{Introduction}

The geological and technological investigation (GTI) into drilling is a toolkit enhancing efficiency of exploratory drilling. In conducting the GTI, drilling parameters are recorded and processed to obtain data on the geological environment. The GTI is applied to determining the boundaries of useful mineral deposits, assessing rock rigidity, improving drilling and borehole charge designs, controlling equipment wear, etc.

Basic obstacles to conducting the GTI are low quality of data and lack of effective methods of the data analysis [1]. Intensive industrial implementation of microprocessor machines enhances quality of data, yet analytical methods of data analysis and interpretation require further investigations. Development of effective methods of analyzing parameters of drilling is quite topical to form a geological structure model.

So far, there have been conducted numerous researches into regularities, mathematical and simulation modeling, formalization of borehole drilling in iron ore mining and processing as well as various methods of operating control of parameters of these processes. It is noteworthy that application of the above methods and means calls for relevant mathematical apparatuses to enable formalization of regularities of interrelations of drilling parameters and characteristics of mineralogical-technological iron ore types making

\footnotetext{
*Corresponding author: vtron@ukr.net
} 
up rocks. To elaborate corresponding mathematical tools, it is necessary to perform a research into mathematical and simulation modeling of the processes occurring in the drilling rig and develop methods of operative measurement and processing of these processes parameters. The obtained results will enable increased efficiency of the technology and methods of borehole drilling control in iron ore mining through applying data on the rock geological structure in the course of drilling.

Formation of a model of the geological structure based on the data on measuring parameters of a drilling rig (drilling rate, torque, energy characteristics) and rock properties studied through determining regularities of changing parameters of the specifically formed ultrasonic field is a promising approach to improving the technology, increasing borehole drilling efficiency in mining iron ore materials presented by several mineralogical and technological types.

\section{Analysis of researches and publications}

At present, various research efforts are being made to create methods of forming a geological structure model in borehole drilling [2 -6]. However, requirements to quality and operability of the data on drilling cannot be fully satisfied because of the technical level and engineering and method potentials of available information and measuring systems and their separate units. Unfortunately, it is quite complicated to model all interactions occurring during drilling [3]. In researches by Xiaowei Pan [7], Victor Mwango Bowa [8] and [14-15] there is an approach to statistic optimization of drilling processes in ore mining and processing, yet little attention is paid to dynamic processes occurring in the course of borehole drilling. Under complicated geological structure conditions of iron ore deposits, this index is not sufficient and there appears a necessity to analyze more variables confirming the research results $[10,16]$.

Determining a rock type during drilling by two indices - mechanical drilling rate and torque suggests in [11]. It is the drawback of the approach to achieve the desired accuracy of rock identification only in the binary geological structure as the indicated parameters are quite accurate to identify homogeneity and strength of rock. With several mineralogical and technological types, this approach is not applicable to assure a required result.

In [12] by Natalie Beattie, to monitor drilling, the following parameters are used: horizontal and vertical vibrations, axis pressure, torque, drilling rate, rotation rate, etc. The disadvantage of this approach is consideration of a drilling rig's parameters as controlled ones while properties of drilled rocks are not measured directly or indirectly. In [13] by Jorge Martin, a similar approach is applied to recognizing the geological structure that incorporates three types. However, like in the previous work, rock types are recognized by drilling rig parameters without measuring characteristics of the drilled rock.

In $[17-20]$, there are offered measuring mining systems based on ultrasonic methods of etermining characteristics of ore materials. [16, 21,24] suggest application of all these methods to recognizing mineralogical ore types. Improved energy-saving technologies of iron ore mining and processing are described in [22, $28-30]$. New approaches to solving the problems of adaptive control and determining stability of their solutions, developing and expanding information and algorithmic support for nonformalized data as well, creating modern adaptive systems of industrial control based on smart technologies are substantiated in $[23,25]$. [26, 27] consider methods of modeling non-linear dynamic objects of concentration based on nuclear operators, formation of optimal control over multi-contour structures based on the Hardy space norms and multi-criteria control of nonlinear dynamic objects with distributed parameters. 


\section{Research aim}

The research is intended to improve the technology and increase efficiency and quality of automated borehole drilling control for mining iron ores of several mineralogical and technological types. This requires developing a method of structural and parametrical identification of the geological structure model of the drilled rock based on indirect data on ultrasonic field parameters formed specifically and changed during drilling together with parameters of a drilling rig (drilling rate, torque, energy characteristics).

\section{Mathematical representation and description of drilling processes}

To assure adequacy of mining objects description, some new mathematical models and new representations of their physical and mechanical characteristics are to be developed and introduced.

It is known from [5] that real rock is a complex conglomerate of amorphous and crystalline compounds of various strength properties, structural composition and granulometric composition of a stochastic nature due to which characteristics of a certain rock are not definite, this fact being confirmed by the same rocks from different deposits. In terms of computation automation and increased qualitative representation of objects' spacious images, it is quite promising to consider meta-mathematics of fuzzy sets that provides the basis for the physical and mechanical characteristic of $\sigma_{c}, \bar{\tau}, f, A_{p}$ presented as fuzzy numbers $s, t, f$ and $a$ respectively and each of them is described by a fuzzy set. Elements of the above sets are pairs of numbers $\left\langle\mu_{i} / U_{i}(x)\right\rangle$ [32], where $\mu_{i}(u)$ and $U_{i}(x)$ are functions of membership and the range of definition (value) of characteristics as subsets of some universal sets $S, T, F$ or $A$ for which $\sigma_{c} \in S, \bar{\tau} \in T, f \in F, A_{p} \in A$.

In this case, a rock is defined as a linguistic variable, a set of basic terms of which includes fuzzy variables built on indicated fuzzy sets [32]. The values of the linguistic variable are determined by the decision making procedure [31, 33].

The set of linguistic variables found via indirect experimental data identifies the research object's profile as a fuzzy ratio $[33,34]$ :

$$
R=\bigcup_{F \cdot A \cdot \mathrm{T} \cdot \Sigma} \mu(f, a, \tau, \sigma) /(F, A, \mathrm{~T}, \Sigma) \cap \bigcup_{Z} \mu(z) /(Z) \cap \bigcup_{\Delta Z} \mu(\Delta z) /(\Delta Z)
$$

under: $(f, a, \tau, \sigma) \in F, A, \mathrm{~T}, \Sigma$, where $\Delta z, Z$ is rock thickness and depth of its occurrence; $\mu(f, a, \tau, \sigma)$ is a subjective extent to which the element $(f, a, \tau, \sigma)$ corresponds to the notion of a certain rock; $\mu(z)$ is a subjective extent to which the element $(z)$ corresponds to the notion of drilling depth; $\mu(\Delta z)$ is a subjective extent to which the element $(\Delta z)$ corresponds to the notion of rock thickness.

The adaptive control of drilling is based on the model composed of four subsystems.

The subsystem of the object's image restoration with the signals $w(t)$ and $\theta(t)$ coming to the subsystem input and the signal $\varphi(t)$ formed at the output. As $\theta(t)$ is the parameter that can determine the closed system condition, the given subsystem is described by the operator influencing $w$ and depending on the $\theta$ parameter: $\phi=H_{\phi w}(\theta) w$.

This subsystem includes the controlled object and the feedback controller (for adaptive 
control) or the closed model of the object when identifying the output error.

The error subsystem with the signals $w(t), \varphi(t)$ and $\theta(t)$ coming to the subsystem input and the error signal $e(t)$ formed at the output to perform adaptive correction. Considering the parametric dependency of $e$ on $\theta$ and applying expression (1) to excluding the dependency on $\varphi$ in its evident form, we have

$$
e=H_{e w}(\theta) w .
$$

The subsystem of data correlation that provides: correlation of data of various mathematical classes and their combining into the generalized space of fuzzy data; introduction of the adaptive control problem into the correction class, determination of the extent and norm of comparing elements of the compact of the input identification area:

$$
w \Leftrightarrow \tilde{w} \Leftrightarrow\|\tilde{w}\|, w \in R ; \quad \tilde{w} \in R \otimes \tilde{R},
$$

where the symbol « » denotes functionality of fuzziness.

The adaptation subsystem with e and $\varphi$ used to obtain evaluation of the $\theta$ parameter. It is evident that in this case, there is no parametric dependency on $\theta$ as it is part of this subsystem condition. The correction algorithm $\theta$ is written as:

$$
\theta=\Omega(e, \phi) .
$$

Formula (3) can also include the parametric dependency $\Omega$ on the step $\varepsilon$ and the initial evaluation of the $\theta$ parameter. This division of the adaptive system into four subsystems is not standard as there is no controlled object here and the error has a more general form than the standard prediction error. Yet, this structure enables focusing on the importance of signal properties in adaptation and general characteristics of operators affecting these signals ignoring the structures and parameters of the controlled object and the controller. Full description of the adaptive system looks like:

$$
\left[\begin{array}{c}
e \\
\phi \\
\varphi
\end{array}\right]=\left[\begin{array}{c}
\tilde{F} \\
K
\end{array}\right] \otimes\left[\begin{array}{c}
H_{e w}(\theta) \\
H_{\phi w}(\theta) \\
F_{\phi w}(\theta)
\end{array}\right] \cdot \tilde{w}=\left[\begin{array}{c}
\tilde{H}_{e w}(\theta) \\
\tilde{H}_{\phi w}(\theta) \\
\tilde{F}_{\phi w}(\theta)
\end{array}\right] \cdot \tilde{w} \equiv H(\theta) \cdot w,
$$

where $\theta=\Omega(e, \phi), \tilde{H}_{*}(\theta), \tilde{w}$ mean both discrete and fuzzy classes of data and functions.

Let the rotating and reciprocating movement of a drilling rig be normally directed to the free surface of the semi-space $Z>0$ filled with the interaction medium and along the whole symmetry axis. It is assumed that the medium is of either isotropic or orthotropic structure and changes of its physical and mechanical properties along the height ( $Z$ coordinate) can be described by the piecewise continuous function with the finite number of first-kind discontinuity points corresponding to coordinates $z_{n}, n=1,2, \ldots, N$; where $N$ is the number of medium layers. Between discontinuity points, the monotonous change of the medium properties is suggested, they are described by strength parameters (compressive resistance, the shearing strength index, the hardness factor according to the Protodiakonov scale, specific fracture energy, etc.). Without losing generality, we assume that $N=2$. Then, each of total components affecting $\mathrm{PO}$ on the part of OC, $M$ and $F$ should include the influence of various strength layers:

$$
\wp_{1} \equiv M=C_{M} \int_{V} d M\left(P(r, z), \sigma(r, z) ; \quad \wp_{2} \equiv F=C_{F} \int_{V} d F(P(r, z), \sigma(r, z),\right.
$$


that can be approximately presented as dependencies:

$$
M \approx C_{M} \int_{S} k_{M} d M(P(r, z), \sigma(r, z)) ; \quad F \approx C_{F} \int_{S} k_{F} d F(P(r, z), \sigma(r, z)) .
$$

Outside the diagonals $z=z^{*}$ and $r=r^{*}$, the functions $M$ and $F$ at any moment $t$ satisfy similar equations like:

$$
L_{M} \wp_{M}(r, \rho ; z, \varsigma)=0, L_{F} \wp_{F}(r, \rho ; z, \varsigma)=0, r \neq r^{*}, z \neq z^{*},\left(z, z^{*}\right) \in \Pi_{1},\left(r, r^{*}\right) \in \Pi_{2},
$$

where $L_{M}, L_{F}$ are linear operators. Let the following inequalities be for parameters of the distinguished layers:

$$
\tau_{1}<\tau_{2} ; \sigma_{1}<\sigma_{2}
$$

where 1 and 2 are the upper and the lower (adjacent) layers of CB respectively. Then, for mode power parameters developed in the working body, the following inequalities are observed:

$$
M_{1}<M_{2} ; F_{1}<F_{2} .
$$

Considering space configuration and contact lines with OC, expressions describing the moment $M$ and the weight capacity $F$ developed on the drilling bit can be written as a sum of integrals, integrand expressions in the first integrals of each equation are determined experimentally and are known:

$$
\begin{aligned}
& \varphi_{M}(z, \bar{\tau})=C_{M}\left[\int_{z}^{z_{0}} K_{M} \bar{\tau}\left(z_{0}-\xi\right) d \xi\right] \\
& \varphi_{F}\left(z, \sigma_{c}\right)=C_{F}\left[\int_{z}^{z_{0}} K_{F} \sigma_{c}\left(z_{0}-\xi\right) d \xi\right],
\end{aligned}
$$

where $K_{M}, K_{F}$ are nuclei of the first and the second integral equations; $\varphi_{M}, \varphi_{F}, C_{M}, C_{F}$ are calculated functions and constants [33, 34].

Then the nuclei $K_{M}, K_{F}$ describing the interaction "working body-environment" are determined through face characteristics $[33,34]$ and equal to

$$
K_{M}=\alpha \xi h(t(\xi)) ; K_{F}=\beta_{0}+\beta h(t(\xi)),
$$

while the expressions for the functions $\varphi_{M}, \varphi_{F}$ are

$$
\varphi_{M}=M_{e f}(t(z))-C_{M} \int_{z_{1}}^{z} \alpha h z \bar{\tau} d z ; \quad \varphi_{F}=F_{e f}(t(z))-C_{F} \int_{z_{1}}^{z}\left(\beta_{0}+\beta h\right) \sigma_{c} d z
$$

In the inverse problem, the observation characteristic (of expected effects) is a function $u \equiv u(x)$.

\section{Algorithm of interpreting experimental data}

Let $N_{j}(t)=U_{0} I_{j}(t)$ be the power consumed by drive motors of the machine (general $L_{1}$ functions). The problem of comparing the functions $N_{j}(t)$ and some desired vector $f(z)$ is reduced to solving two problems: the time problem (determining effective solutions of values of strength parameters $f, \bar{\tau}, \sigma_{c}, \sigma_{p}$, specific instrument feed $h(t)$ and drilling 
depth $z(t)$; the space problem (restoring real values of strength parameters according to their efficiency).

The time problem $[33,34]$ contrasts the function $\mathrm{W}^{*}(\mathrm{t})$ determined as effective specific power intensity of drilling to the vector $f \equiv\left\{f, \bar{\tau}, \sigma_{c}, \sigma_{p}\right\}$ :

$$
W^{*}(t)=\int_{t_{0}}^{t}\left\{\left[F\left(f^{*}(t)\right) h(t)+M\left(f^{*}(t)\right)\right] \omega+A_{y} n_{y}\right\} d \tau,
$$

thus determining the implicit nonlinear operator $A(H, t) \equiv W^{\prime}(t)$ with the proximity measure of $N_{j}$ and $W^{*}$ :

$$
\rho\left(N, W^{*}\right)=\|A-N\|_{L_{1}}, N=\sum N_{j} .
$$

Let $H$ be thickness of the drilled rock layer, $H=z(t)$. Then, the solution of the first problem is reduced to the following. If

$$
F^{*}[H]=\|A-N\|_{L_{1}}
$$

is the functional determined according to $H>0$, one should determine the values of $f^{*}(t), h(t), z(t)$, minimizing this functional under: (1) the monotonous solution $f^{*}(t)>0 ;(2)\|A-N\|_{L_{1}} \leq \varepsilon\left(\delta_{I}\right)$.

The space problem $[33,34]$ contrasts the function $W(z)$ determining the real specific power intensity of rock crushing by the given drilling method to the segment $\left[z, z_{0}\right], 0 \leq z_{0}-$ $z \leq h_{i}$ where $Z_{0}$ and $h_{i}$ are the value of the current coordinate of the top of the rock-crushing bit and the height of its working surface respectively:

$$
W(z)=\int_{z_{0}}^{z}\left\{\left[F(f) H(t(\zeta))+M\left(\tau_{c p}\right)\right] \frac{\omega(t(\zeta))}{V(t(\zeta))}+A_{y}(\sigma) n_{y} / \eta\right\} d \zeta,
$$

thus determining the nonlinear operator $B\left(z_{0}, z\right)=W(z)$ with the proximity measure of $B$ and $W^{*}$ :

$$
\rho\left(B, W^{*}\right)=\left\|B-W^{*}\right\|_{L_{1}} .
$$

In this case, the solution of the second problem is reduced to the following. If

$$
F\left[z_{0}-z\right]=\left\|B-W^{*}\right\|_{L_{1}}
$$

is the functional determined on the section $\left[0, z_{0}-z\right], f(z)$, there should be determined $\bar{\tau}(z), \sigma_{c}(z), \sigma_{p}(z), A_{p}(z)$ and $l_{n}$ minimizing this functional under:

$$
\left\|B-W^{*}\right\|_{L_{1}} \leq \varepsilon(f)
$$

and limitations of the solutions:

$$
0<f<f_{\max } ; 0<\bar{\tau}<\tau_{\max } ; 0<\sigma_{c}<\sigma_{\max }, 0<\sigma_{p}<\sigma_{\max }, z_{0}-z \leq h_{i},
$$

while $z(t)$ satisfies the homeomorphism condition.

Auxiliary information on solution of the given problem can be obtained by assessing physical and mechanical characteristics of ferromagnetic rocks through ultrasonic measurements. The given problem is solved by forming a constant magnetic field and 
electromagnetic pulses of sinusoidal oscillations of the desired amplitude and duration while drilling boreholes in the rock massif in the measurement area. The frequency of their filling is changed within certain limits determining dependency of the amplitude on the frequency of formed elastic oscillations, which, in turn, provides the data on characteristics of ferromagnetic rocks and structure of their occurrence. To excite elastic oscillations in ferromagnetic rocks of the rock massif, the acoustic electromagnetic transducer is employed. It is characterized by a distributed structure composed of a constant magnetic field source, an alternating magnetic field source and some volume of a ferromagnetic component of the rock in which the processes of transformation of the electromagnetic field power into that of elastic oscillations of medium particles occur.

While exciting elastic disturbances, the electromagnetic transducer transforms electromagnetic signals into elastic oscillations of ferromagnetic rocks via the magnetostrictive effect and the ponderomotive action of the electromagnetic field. In some point of the ferromagnetic material with the coordinates $x_{k}$, the constant magnetic field of $H^{0}\left(x_{k}\right)$ intensity and the alternating magnetic field of $H^{0}\left(x_{k}, t\right)$ intensity at the moment $t$ are formed. Within the existing magnetic fields $H^{*}\left(x_{k}, t\right)$ and $H^{0}\left(x_{k}\right)$, there are formed deformations of the ferromagnetic material. Elastic waves transfer the power further in the deformation zone. Thus, the acoustic electromagnetic transducer creates elastic oscillations right in the zone of measuring characteristics of ferromagnetic rocks of the rock massif. As there are no intermediate elements to transfer formed elastic oscillations into the medium, the errors of measuring its characteristics determined by these factors are also absent.

The amplitude and frequency of elastic acoustic oscillations depend on the content and structure of distribution of the ferromagnetic component inside the rock, physical and mechanical characteristics and the rock massif condition. The receiver of acoustic oscillations receives the formed signal in the rock massif. The amplitude of the received signal characterizes the amplitude value of the ferromagnetic component content in the measuring zone: the greater the concentration of the ferromagnetic material is, the higher the efficiency of transformation of the electromagnetic pulse power into the mechanical power of rock deformation and, consequently, the amplitude of elastic oscillations formed in the rock massif are.

The electromagnetic acoustic transducer and receiver are mounted on the working body of the drilling rig that moves along the borehole with the known velocity. To assess the average content of the ferromagnetic component in the controlled area of the rock massif, the average value of the amplitude $A_{a v}$ of the elastic oscillations formed and received by the receiver is measured. In the course of forming elastic oscillations in the ferromagnetic rock, their reflection, interference and absorption take place. Parameters of these processes depend on the amplitude and frequency of the electromagnetic pulse, the structure of distribution of the ferromagnetic component inside the rock massif and physical and mechanical properties of rocks. Reflection of elastic oscillations on the structural irregularities of ferromagnetic component distribution inside the rock and interference of reflected signals cause varied frequency of resulting elastic oscillations. The value of these variations is determined by the ratio of the wave length of the formed elastic oscillations and the size of impregnations of the ferromagnetic component in the rock, i.e. the structure of its distribution inside the rock massif. Changes of the frequency of sinusoid oscillations filling the electromagnetic pulse cause changes of the wave length of elastic oscillations formed in the rock massif and conditions of their propagation in the measuring zone respectively.

Dependency of reflection and interference of formed elastic oscillations in rocks on the size and structure of irregularities causes changes of the received signal amplitude. Changed frequency of sinusoid oscillations that fill the electromagnetic pulse enables forming elastic oscillations with various wave lengths, thus detecting and assessing the reaction of the rock massif to the ratio of this parameter, size and structure of the 
ferromagnetic component distribution. Thus, dependency of the amplitude on distribution of resulting elastic oscillations formed in the rock massif is conditioned by the content and distribution structure of the ferromagnetic component inside the rock massif as well as by physical and mechanical properties of rocks. Similar preliminary measurements of reference samples are made to identify mineralogical types of the ferromagnetic rock under study on the basis of obtained measurement results.

The applied method enables obtaining auxiliary information on concentration and structure of the ferromagnetic component in the rock, i.e. its mineralogical type. There is no need to use any borehole liquid or special devices of introducing elastic oscillations into the rock massif, this expanding the area of the method application. The mineralogical types presented in Table 1 were considered [5].

Table 1. Percentage ratio of data on rock types.

\begin{tabular}{|c|c|c|c|c|c|c|}
\hline \multirow{2}{*}{ Ore type } & \multicolumn{5}{|c|}{ Percentage, $\%$} & \multirow{2}{*}{$\begin{array}{c}\text { Total } \\
\text { data ratio }\end{array}$} \\
\hline & Quarts & Magnetite & Martite & Hematite & Siderite & \\
\hline $\begin{array}{l}1 \text { - magnetite } \\
\text { hornstones; }\end{array}$ & 63.7 & 30.9 & 0 & 1.4 & 3.8 & $25.6 \%$ \\
\hline $\begin{array}{l}2 \text { - silicate-carbonate- } \\
\text { magnetite hornstones; }\end{array}$ & 68.4 & 21.7 & 0 & 0.4 & 9.1 & $15.14 \%$ \\
\hline $\begin{array}{l}3 \text {-jaspilite- magnetite } \\
\text { and hematite-magnetite } \\
\text { hornstones; }\end{array}$ & 64.5 & 30.2 & 0 & 1.5 & 3.8 & $17.8 \%$ \\
\hline $\begin{array}{l}4 \text { - semi-oxidized and } \\
\text { oxidized hornstones; }\end{array}$ & 65.4 & 24.4 & 3.3 & 3.7 & 3.2 & $13.5 \%$ \\
\hline $\begin{array}{l}5 \text { - silicate shales, barren } \\
\text { hornstones and quarts }\end{array}$ & 74.6 & 4.5 & 0 & 0.7 & 20.2 & $27.96 \%$ \\
\hline
\end{tabular}

Table 2 contains the research results.

Table 2. Accuracy of identifying the ore types with varied quantities of input data parameters.

\begin{tabular}{|c|c|c|c|c|c|c|}
\hline \multirow{2}{*}{ Number of inputs } & \multicolumn{5}{|c|}{ Ore type } & \multirow{2}{*}{ Accuracy } \\
\cline { 2 - 5 } & 1 & 2 & 3 & 4 & 5 & \\
\hline 2 & $45.71 \%$ & $14.42 \%$ & $16.23 \%$ & $10.42 \%$ & $51.38 \%$ & $54.78 \%$ \\
\hline 3 & $67.54 \%$ & $38.32 \%$ & $45.03 \%$ & $32.53 \%$ & $74.32 \%$ & $68.03 \%$ \\
\hline 5 (Vibrations) & $72.63 \%$ & $48.67 \%$ & $62.56 \%$ & $38.32 \%$ & $81.54 \%$ & $73.65 \%$ \\
\hline 5 (Deviations) & $76.24 \%$ & $53.96 \%$ & $76.54 \%$ & $43.70 \%$ & $86.34 \%$ & $77.36 \%$ \\
\hline 7 & $92.54 \%$ & $69.56 \%$ & $83.05 \%$ & $67.32 \%$ & $94.52 \%$ & $83.71 \%$ \\
\hline 8 & $97.78 \%$ & $83.23 \%$ & $93.12 \%$ & $81.63 \%$ & $98.94 \%$ & $90.94 \%$ \\
\hline
\end{tabular}

For the binary structure like ore or gangue, the drilling rate and torque are input parameters sufficient to provide efficient evaluation with accuracy of identification of up to $92 \%$ [2]. Conducted researches indicate that it is not sufficient for iron ore materials. Accuracy of identification in case of two inputs makes $54.78 \%$, seven inputs provide $83.71 \%$, and eight inputs provide $90.94 \%$.

\section{Conclusions}

Further development of the mathematical adaptive control theory is suggested that implies adaptive control performed for the data space not generally subject to formalization and characterized by the uncontrolled error. The obtained results are used in solving the problem of the drilling adaptive control for which the data on the controlled object is indirect, while according to the target function, decision making is based on restoration 
(within the framework of solving the incorrect problem) of the object's "image"; the value of the object's parameters and input data are not formalized in the conventional sense and, under data uncertainty and insufficiency, are fuzzy numbers, linguistic variables, etc.

The research results indicate that identification of the geological structure will be successful only if the maximum number of data on drilling will be used and the training set will include the full amount of data on all mineral formations that can occur during drilling a borehole.

The authors express their sincere gratitude to Kryvyi Rih National University for support in conducting research.

\section{References}

1. Smith, L.I. (2002). A tutorial on principal component analysis. URL: http://www.cs.otago.ac.nz/cosc453/student tutorials/principal components.pdf

2. Martin, J. (2007). Application of pattern recognition techniques to monitoring-while-drilling on a rotary electric blast hole drill at an open-pit coal mine. Master's thesis. Kingston: Queen's University.

3. Liu, H., \& Yin, K. (2001). Using information extracted from drill data to improve blasting design and fragmentation. Fragblast, 5(3), 157-179. https://doi.org/10.1076/frag.5.3.157.7384

4. Beattie, N. (2009). Monitoring-while-drilling for open-pit mining in a hard rock environment. Master's thesis, Queen's University.

5. Kozin, V.Z. (2008). Issledovanie rud na obogatimost'. Ekaterinburg: Ural'skiy gosudarstvennyy gornyy universitet.

6. Demuth, H., Beale, M., \& Hagan, M. (2008). Neural Network Toolbox 6: User's Guide. Natwick, MA: The MathWorks Inc.

7. Xiaowei, P. (2012). Optimization of mineral processing plant through ROM ore size. Akademia Górniczo-Hutnicza Journal of Mining and Geoengineering, 36(4), 123-132.

8. Mwango Bowa, V. (2015). Optimization of blasting design parameters on open pit bench a case study of Nchanga open pits. International Journal of Scientific \& Technology Research, 4(9), 45-51.

9. Yue, Z.Q., Lee, C.F., Law, K.T., \& Tham L.G. (2015). Automatic monitoring of rotary percussive drilling for ground characterization. International Journal of Rock Mechanics \& Mining Sciences, (41), 573-612. https://doi.org/10.1016/j.ijrmms.2003.12.151

10. Schunnesson, H. (1998). Rock characterisation using percussive drilling. International Journal of Rock Mechanics \& Mining Sciences, 35(6), 711-725. https://doi.org/10.1016/s01489062(97)00332-x

11. Schunnesson, H., \& Holme K. (1997). Drill monitoring for geological mine planning in the Viscaria copper mine, Sweden. Canadian Mining and Metallurgical Bulletin, 90(1013), 83-89.

12. Beattie, N. (2012). Monitoring-while-drilling for open-pit mining in a hard rock environment: Master's thesis. Kingston: Queen's University.

13. Martin, J. (2013). Application of pattern recognition techniques to monitoring-while-drilling on a rotary electric blast hole drill at an open-pit coal mine. Master's thesis. Kingston: Queen's University.

14. Segui, J.B., \& Higgins M. (2012). Blast design using measurement while drilling parameters. Fragblast: International Journal for Blasting and Fragmentation, 6(3-4), 287-299. https://doi.org/10.1076/frag.6.3.287.14052

15. Scoble, M. J., Peck, J., \& Hendricks, C. (1989). Correlation between rotary drill performance parameters and borehole geophysical logging. Mining Science and Technology, (8), 301-312. https://doi.org/10.1016/s0167-9031(89)90448-9

16. Morkun, V., \& Tron, V. (2014). Automation of iron ore raw materials beneficiation with the operational recognition of its varieties in process streams. Metallurgical and Mining Industry, (6), 4-7. 
17. Morkun, V., Morkun, N., \& Pikilnyak, A. (2015). The study of volume ultrasonic waves propagation in the gas-containing iron ore pulp. Ultrasonics, (56), 340-343. https://doi.org/10.1016/j.ultras.2014.08.022

18. Morkun, V., Morkun, N., \& Pikilnyak, A. (2014). Ultrasonic facilities for the ground materials characteristics control. Metallurgical and Mining Industry, (2), 31-35.

19. Morkun, V., Morkun, N., \& Pikilnyak, A. (2014). Simulation of high-energy ultrasound propagation in heterogeneous medium using k-space method. Metallurgical and Mining Industry, (3), 23-27.

20. Morkun, V., Morkun, N., \& Pikilnyak, A. (2014). Simulation of the Lamb waves propagation on the plate which contacts with gas containing iron ore pulp in Waveform Revealer toolbox. Metallurgical and Mining Industry, (5), 16-19.

21. Morkun, V., Tron, V., \& Goncharov, S. (2015). Automation of the ore varieties recognition process in the technological process streams based on the dynamic effects of high-energy ultrasound. Metallurgical and Mining Industry, (2), 31-34.

22. Morkun, V., Morkun, N., \& Pikilnyak, A. (2014). Ultrasonic phased array parameters determination for the gas bubble size distribution control formation in the iron ore flotation. Metallurgical and Mining Industry, (3), 28-31.

23. Morkun, V., \& Tron, V. (2014). Ore preparation energy-efficient automated control multi-criteria formation with considering of ecological and economic factors. Metallurgical and Mining Industry, (5), 8-10.

24. Morkun V., \& Tcvirkun S. (2014) Investigation of methods of fuzzy clustering for determining ore types. Metallurgical and Mining Industry, (5), 11-14.

25. Morkun, V., Morkun, N., \& Pikilnyak, A. (2014). The gas bubble size distribution control formation in the flotation process. Metallurgical and Mining Industry, (4), 42-45.

26. Morkun, V., Morkun, N., \& Pikilnyak, A. (2015). Adaptive control system of ore beneficiation process based on Kaczmarz projection algorithm. Metallurgical and Mining Industry, (2), 35-38.

27. Morkun, V., Morkun, N., \& Tron, V. (2015). Formalization and frequency analysis of robust control of ore beneficiation technological processes under parametric uncertainty. Metallurgical and Mining Industry, (5), 7-11.

28. Golik V., Komashchenko V., \& Morkun V. (2015). Geomechanical terms of use of the mill tailings for preparation, Metallurgical and Mining Industry, (4), 321-324.

29. Golik, V., Mitsik, M., Morkun, V., Morkun, N., \& Tron, V. (2019). Transportation of concentration and leaching tailings in underground mining of metal deposits. Mining of Mineral Deposits, 13(2), 111-120. https://doi.org/10.33271/mining13.02.111

30. Golik, V., Komashchenko, V., Morkun, V., \& Burdzieva, O. (2015). Metal deposits combined development experience. Metallurgical and Mining Industry, (6), 591-594.

31. Golik, V., Komaschenko, V., Morkun, V., \& Khasheva, Z. (2015). The effectiveness of combining the stages of ore fields development. Metallurgical and Mining Industry, (5), 401-405.

32. Borisov, A.N., Alekseev, A.V., \& Merkuryeva G.V. (1989). Obrabotka nechetkoy informatsii v sistemakh prinyatiya resheniy. Moskva: Nauka.

33. Zade, Z. (1972). Ponyatie lingvisticheskoy peremennoy i ee prilozhenie. Moskva: Mir.

34. Morkun, V.S., Tsokurenko, A.A., \& Lutsenko, I.A. (2005). Adaptivnye sistemy optimalnogo upravleniya tekhnologicheskimi protsessami. Kryvyi Rih: Mineral.

35. Tsokurenko, A.A. (1998). Informatsionno-vychislitelnyy kompleks i obratnye zadachi. Kyiv: Naukova dumka.

36. Tikhonov, A.N., \& Arsenin, V.Ya. (1986). Metody resheniya nekorrektnykh zadach. Moskva: Nauka. 